\title{
Tsallis mapping in growing complex networks with fitness
}

\author{
Guifeng $\mathrm{Su}^{1}$, Xiaobing Zhang ${ }^{2}$, Yi Zhang ${ }^{1 *}$ \\ 1 Department of Physics, Shanghai Normal University, \\ Shanghai 200230, People's Republic of China \\ 2 School of Physics, Nankai University, \\ Tianjin 300371, People's Republic of China
}

\begin{abstract}
We introduce Tsallis mapping in Bianconi-Barabási (B-B) fitness model of growing networks. This mapping addresses the dynamical behavior of the fitness model within the framework of nonextensive statistics mechanics, which is characterized by a dimensionless nonextensivity parameter $q$. It is found that this new phenomenological parameter plays an important role in the evolution of networks: the underlying evolving networks may undergo a different phases depending on the $q$ exponents, comparing to the original B-B fitness model, and the corresponding critical transition temperature $T_{C}$ is also identified.
\end{abstract}

*Electronic address: yizhang@shnu.edu.cn 


\section{INTRODUCTION}

The seminal work by Watts and Strogatz on small-world networks [1], and that by Barabási and Albert on scale-free networks (i.e., B-A networks) [2], have pioneered a wave of research activities on the complex networks in last decade (see, for instance, recent review articles [3 [5], books [6, 7], and references therein). Research toward understanding the new critical phenomena in complex networks, e.g., the structural phase transitions in networks, is also a rapidly developing area [8]. It is found that the condensation phase transitions occur in many complex network models [9 22]. Particularly, in Ref. [11], Bianconi-Barabási (B-B) fitness model [12] was mapped into an equilibrium Bose gas, a Bose-Einstein condensation (BEC) behavior was obtained, and the critical point for condensation was identified by mean-field arguments. There the well-known B-A networks [2] are generalized by introducing the fitness parameter, $\eta_{i}$. At each time step, a new node with $m$ links is added to the nodes that already presented in the network. The fitness $\eta_{i}$, accounting for the competition for links in the network, is chosen randomly from a distribution. The probability $\Pi_{i}$ that a new node connects one of its $m$ links to a node $i$ depends on the number of links, $k_{i}(t)$, and on its fitness $\eta_{i}$, is

$$
\Pi_{i}=\frac{\eta_{i} k_{i}}{\sum_{j} \eta_{j} k_{j}} .
$$

In order to map the fitness model into Bose gases, one assigns an "energy" $\varepsilon_{i}$, introduced randomly from a distribution $g(\varepsilon)$, for the $i$ th node, and the fitness of the node, $\eta_{i}$, is defined as

$$
\eta_{i}=e^{-\beta \varepsilon_{i}}
$$

where the parameter $\beta$, can be identified as inverse "temperature" $\beta=1 / T$. This growing network can be solved in a mean-field approximation and a "chemical potential" $\mu$ is determined by the self-consistent equation

$$
I(\beta, \mu)=\int d \varepsilon g(\varepsilon) n(\varepsilon)=1
$$

where the Bose occupation number $n(\varepsilon)=1 /\left(e^{\beta(\varepsilon-\mu)}-1\right)$ is the number of links attached by the preferential attachment mechanism to nodes with "energy" $\varepsilon$. A BEC phase transition occurs in the networks if $I(\beta, 0)<1$, i.e., the self-consistent equation (3) has no solution (in such case the self-consistent approach fails). A necessary condition for condensation is 
that the distribution $g(\varepsilon) \rightarrow 0$ for $\varepsilon \rightarrow 0$. For this type distributions there exists a critical temperature $T_{c}=1 / \beta_{c}$ such that $I(\beta, 0)<1$ for $T<T_{c}$.

However, it should be noted that BE statistics appearing in B-B growing network is only formally equivalent to that in ideal Bose gases. This is due to the fact that the evolution of B-B network is in essential irreversible and out-of-equilibrium, while the BEC of ideal Bose gases is a temperature-driven equilibrium process. On the other hand, it was argued [23] a wide variety of complex networks have self-similar, fractal property. Under such circumstances, a particularly useful and appropriate framework to describe the dynamical evolution of the network is so-called Tsallis nonextensive statistical mechanics [24-26]. It is a certain generalization of the usual Boltzmann-Gibbs (BG) formulation. For the applications of the formulation in many fields, including complex networks, one may see, e.g., Refs. [27, 28] and references therein. In present work, we consider the possibility of embedding the B-B fitness model in the framework of Tsallis formulation of the non-extensive statistical mechanics. The paper is organized as follows: in Sec. II, we briefly review Tsallis formulation of the nonextensive statistical mechanics, and introduce Tsallis mapping in the B-B fitness model of the complex network. We show that the corresponding rate equation has a power-law solution under some approximation within the high temperature regime, in thermodynamic limit. For general situations without the thermodynamic limit, the numerical simulations are need. The main simulation results are shown and discussed in Sec. III. Finally, we summarize and draw our conclusions.

\section{TSALLIS MAPPING IN B-B FITNESS NETWORKS}

In Tsallis formulation of non-extensive statistical mechanics, the central equation is socalled the nonextensive entropy, which is defined as

$$
S_{q}=\frac{1}{q-1}\left(1-\sum_{i} p_{i}^{q}\right),
$$

where $p_{i}$ is the probabilities for the ensemble to be in the state $i$. The non-extensive parameter $q$ describes the degree of deviations of a physical quantity from extensive and therefore may be regarded as a new phenomenological parameter of the model used, different values of $q-1$ quantifies the departure from the BG limit. The standard results of BG statistical mechanics are recovered in the limit $q \rightarrow 1$. The application of the non-extensive 
statistical mechanics to Boson gases predicts a $q$-deformed BE statistics [25]:

$$
n_{q}(\varepsilon)=\frac{1}{e_{q}^{\beta(\varepsilon-\mu)}-1},
$$

where $n_{q}(\varepsilon)$ is the occupation number of an energy level $\varepsilon$ and $\mu$ the chemical potential. The parameter $\beta$ is the inverse temperature. A $q$-exponential function is introduced in Eqn. (5) as follows [29]:

$$
e_{q}^{z} \equiv[1+(1-q) z]^{1 /(1-q)}
$$

if the argument $1+(1-q) z$ is positive, $e_{q}^{z}$ equals to zero otherwise. Its inverse function, the $q$-logarithm, for any positive real number $z \in \mathbb{R}^{+}$, is

$$
\ln _{q} z \equiv \frac{z^{1-q}-1}{1-q}
$$

Both $q$-logarithm and $q$-exponential functions go back to the usual logarithm and exponential functions, respectively, when one takes $q \rightarrow 1$ limit.

In order to map B-B fitness model to a boson gas with Tsallis statistics, under the selfconsistency condition, we assign the $i$ th node with a fitness, $\eta_{i}$, by

$$
\eta_{i}=e_{q}^{-\beta \varepsilon_{i}}=\left(1+(1-q)\left(-\beta \varepsilon_{i}\right)\right)^{1 /(1-q)},
$$

and its inverse is

$$
\varepsilon_{i}=-\frac{1}{\beta} \ln _{q} \eta_{i}=-\frac{1}{\beta} \frac{\eta_{i}^{1-q}-1}{1-q},
$$

where $\varepsilon_{i}$ follows the energy level distribution $g(\varepsilon)=C \varepsilon^{\theta}$ and $C$ is a normalization factor. A necessary condition for condensation is that when $\varepsilon \rightarrow 0, g(\varepsilon) \rightarrow 0$ consistently. The resulting rate equation which describes the degree of connectivity at time $t_{i}$ of the $i$ th node (energy level), $k_{i}\left(\varepsilon_{i}, t, t_{i}\right)$, is then:

$$
\frac{\partial k_{i}\left(\varepsilon_{i}, t, t_{i}\right)}{\partial t}=\frac{e_{q}^{-\beta \varepsilon_{i}} k_{i}\left(\varepsilon_{i}, t, t_{i}\right)}{t Z_{t}^{q}}
$$

where

$$
Z_{t}^{q}=\frac{1}{m t} \sum_{j=1}^{t} e_{q}^{-\beta \varepsilon_{j}} k_{j}\left(\varepsilon_{j}, t, t_{j}\right),
$$

is the $q$-deformed partition function. Following the standard statistical mechanics [30], the definition of fugacity $z$ is given, in a consistent way, by $q$-deformed form, $z^{-1}=e_{q}^{-\beta \mu}$, in which $\mu$ is the chemical potential. 
The phase structure of this B-B fitness model with Tsallis mapping is now controlled by two parameters, i.e., the inverse temperature $\beta$ and the nonextensive parameter $q$, instead of (only) one parameter $\beta$ in the original B-B fitness model. These two parameters both affect the phase transition of the network. A few limiting situations are worthy of being discussed before further detailed numerical simulations. First, in $q \rightarrow 1$ limit, the B-B model with Tsallis mapping goes back to the original one with Bose gas mapping, due to the fact that Tsallis $q$-deformed expressions approach to the usual ones, and all the known results of the BB model are recovered as they should. Henceforth, the scale-free phase in original B-B fitness model is trivially recovered when all nodes have the same fitness, i.e., $g(\varepsilon) \sim \delta(\varepsilon)[2$, 11]. Second, with $q \rightarrow 1$ limit and the thermodynamic limit $t \rightarrow \infty$ as well, we find that the rate equation (10) has a power law solution under some appropriate approximation, within high temperature region, in which $\beta$ is a small quantity. This phase, as matter of fact, is similar to the fit-get-rich (FGR) phase in the original B-B fitness model [12].

To see the latter, we assume that the solution takes the following form:

$$
k_{i}\left(\varepsilon_{i}, t, t_{i}\right) \sim\left(\frac{t}{t_{i}}\right)^{f_{q}\left(\varepsilon_{i}\right)}
$$

where $f_{q}(\varepsilon)$ is some unknown power-law exponent and to be determined later. For simplicity we will write $f_{q}(\varepsilon) \equiv f_{q}$ from now on. Taking the energy distribution of the nodes $g(\varepsilon)$ into account, the partition function becomes

$$
\overline{Z_{t}^{q}} \sim \int d \varepsilon g(\varepsilon) \int_{1}^{t} \frac{d t_{0}}{t} e_{q}^{-\beta \varepsilon} k_{i}\left(\varepsilon, t, t_{0}\right),
$$

here we use $\overline{Z_{t}^{q}}$ stand for the partition function averaging over $g(\varepsilon)$. Now inserting $k_{i}$ (in Eqn.(12)) into $\overline{Z_{t}^{q}}$, one obtains

$$
\begin{aligned}
\overline{Z_{t}^{q}} & \sim \int d \varepsilon g(\varepsilon) \int_{1}^{t} \frac{d t_{0}}{t} e_{q}^{-\beta \varepsilon}\left(\frac{t}{t_{0}}\right)^{f_{q}} \\
& =\int d \varepsilon g(\varepsilon) e_{q}^{-\beta \varepsilon} t^{f_{q}-1}\left(\frac{t^{1-f_{q}}-1}{1-f_{q}}\right) \\
& =\int d \varepsilon g(\varepsilon) e_{q}^{-\beta \varepsilon} t^{f_{q}-1}\left(\ln _{f_{q}} t\right) \\
& \sim z^{-1}\left(1-t^{-\left(1-f_{q}\right)}\right)
\end{aligned}
$$

where $\ln _{f_{q}} t$ is the $f_{q}$-logarithm as introduced in Eqn. (7) and the inverse fugacity $z^{-1}$ is

$$
z^{-1}=\int d \varepsilon g(\varepsilon) \frac{e_{q}^{-\beta \varepsilon}}{1-f_{q}} .
$$


Note that in Eqn. (14), $1-f_{q}>0$, such that in thermodynamic limit $t \rightarrow \infty, t^{-\left(1-f_{q}\right)} \rightarrow$ 0 , and hence $\overline{Z_{t}^{q}} \sim z^{-1}$ in the corresponding limit. This makes one able to determine aforementioned power-law exponent $f_{q}$. Note that in high temperature region, $\beta$ is small, and that $q$ is very close to unity (such that $q-1$ is small as well), so we can take the approximation $e_{q}^{-\beta \varepsilon} / e_{q}^{-\beta \mu} \approx e_{q}^{-\beta(\varepsilon-\mu)}$ [31], as a result, the exponent $f_{q}$ of the power-law solution in Eqn. (12) is,

$$
f_{q}=e_{q}^{-\beta(\varepsilon-\mu)},
$$

in $q \rightarrow 1$ limit, this exponent goes back to the one in the original B-B fitness model [11]. The fugacity becomes

$$
z^{-1}=\lim _{t \rightarrow \infty} \overline{Z_{t}^{q}}=\int d \varepsilon g(\varepsilon) \frac{e_{q}^{-\beta \varepsilon}}{1-f_{q}(\varepsilon)}
$$

and the chemical potential $\mu$ is the solution of the following equation,

$$
I_{q}(\beta, \mu)=\int d \varepsilon g(\varepsilon) \frac{1}{e_{q}^{\beta(\varepsilon-\mu)}-1}=1,
$$

which indicates a mapping to Bose gas with Tsallis statistics, and the corresponding occupation number of a energy level $\varepsilon$ is $n(\varepsilon)=\left(e_{q}^{\beta(\varepsilon-\mu)}-1\right)^{-1}$. This just follows Tsallis statistics.

For general situations without taking into account above limits, one has to resort to numerical simulations for current model with Tsallis mapping.

\section{NUMERICAL SIMULATION RESULTS AND DISCUSSIONS}

In the original B-B fitness model, the signal of the condensation phase transition lies on the change of sign of the chemical potential $\mu$, i.e., when $\mu$ experiences a change from negative value to positive value within some regime, that change identifies the critical point of the corresponding phase transition. Similarly, we use the same criterion for the phase transition in the B-B fitness network with Tsallis mapping. This is done by numerically computation of the chemical potential $\mu$, without the thermodynamic limit, according to the following way:

$$
\mu=-\frac{1}{\beta} \ln _{q} \overline{Z_{t}^{q}}=-\frac{1}{\beta} \frac{\left(\overline{Z_{t}^{q}}\right)^{1-q}-1}{1-q},
$$

where $\ln _{q} \overline{Z_{t}^{q}}$ is the $q$-logarithm of $\overline{Z_{t}^{q}}$ which is defined in Eqn. (11). Note that in above numerical simulations, the computation of $\mu$ strictly follows its definition and has no approximations in them. 
The "phase diagrams" of the B-B fitness network with Tsallis mapping are demonstrated by our numerical simulations and are shown in Fig. 1 - 3. In both figures, the size of the evolving network, or the time steps, is taken to be $t=10^{3}$ and we fix $m=2$. In Fig. 1, the absolute value of the chemical potential $\mu$, which is defined in Eqn. (19), is numerically computed with an average over 300 runs, using the energy level distribution $g(\varepsilon)=2 \varepsilon$ for energies $\varepsilon \in(0,1)$, i.e., $\theta=1$ and $C=2$, and for $q=1.0,1.1,1.3$, and 1.5 , respectively. As expected, for $q=1.0$, the phases emerge in the system are coincident with those in the original B-B fitness model: the FGR phase and the BEC phase (the solid line in Fig. 11), and the same transition temperature is located at $T_{B E} \sim 0.8$ (the vertical dash-line on the right). However, when the value of parameter $q$ deviates from 1 , e.g., for $q=1.1,1.3$, and/or 1.5, the phases correspondingly change depending on both the specific value of $q$ and the temperature they take (see the dot-dashed line in Fig. 1). Also, it can be seen from the figure that the transition temperature $T_{c}^{q}$ decreases and $T_{c}^{q} \sim 0.67$ (the vertical dashed line on the left in Fig. 1). In addition, in $T<T_{c}^{q}$ regime, the whole "Tsallis condensate" curve deviates from the BEC curve. On the other hand, it is no surprise that the effect of $q$ parameter disappears gradually in the high temperature limit. The effect of different energy level distribution is shown in Fig. 2, with $\theta=1 / 2$. We also plot the fraction of the total number of links connected to the most connected (or "winner") node, $k_{\max } / m t$, as a function of $T$ (lower panel in Fig. 1 and Fig. 2).

We also plot in Fig. 3 the connectivity degree distribution of the B-B fitness network with Tsallis mapping, i.e., the probability distribution $P(k)$ vs. the connectivity degree $k$, for different values of parameter $q$ and temperatures $T$. In the figure, $P(k)$ is plotted for $g(\varepsilon) \sim \varepsilon$, with nonextensive parameter $q=1.0,1.3$, and 1.5, and with temperature $T=0.08$, $T=0.4, T=0.8$, and $T=4.0$, respectively. Since the critical temperature is $T_{C}=0.67$, in the latter cases (i.e., $T=0.8$ and $T=4.0$ ), the temperature $T>T_{C}$. As we expected, the Tsallis mapping gives the power-law degree distribution for $T>T_{C}, P(k) \sim k^{-\gamma}$, with $\gamma \sim f_{q}$ (see the lower panels in Fig 3 , in which $T=0.8$ and $T=4.0$, respectively). Note that, first, the nonextensive parameter $q$ controls the slope of $P(k)$ at given temperature; second, data points with $q=1$ (open squares with blue color in Fig. 3) in fact correspond to the FGR phase in the original B-B model for $T>T_{C}$. When the temperature decreases, for example, at the critical point $T_{C} \approx 0.67$, the chemical potential changes its sign from positive to negative, and this correspondes to the situation that a condensate phase starts to 

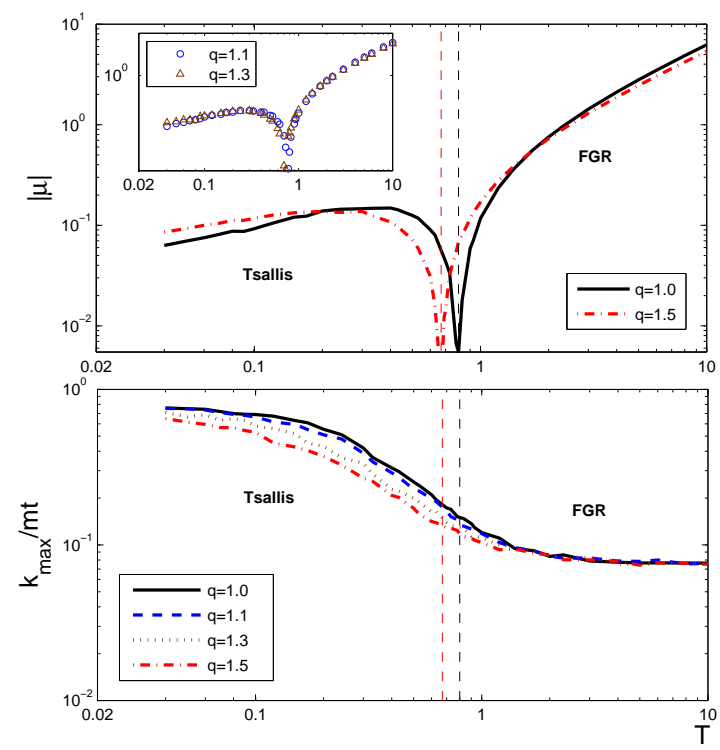

FIG. 1: Numerical simulations of the fitness model with Tsallis mapping for growing complex networks. An energy level distribution $g(\varepsilon) \sim \varepsilon$ is taken in the figure. We set evolving times (system size) to be $t=10^{3}$, and averaged over 300 runs. Upper Panel: Numerically calculated chemical potential using the energies distribution with $\varepsilon \in(0,1)$. The temperatures at which $\mu$ changes sign correspond to the sharp drop in $|\mu|$ on the figure, and identify the different critical temperatures $T_{c}^{q}$ (two vertical dashed lines in the figure) for Tsallis condensation phase with different $q$-value: $q=1.0$ (solid line), corresponds to the results of B-B fitness model; $q=1.5$ (dot-dashed line). The inset plot shows the chemical potential for another choices of $q$ in between: $q=1.1$ (open circles), and $q=1.3$ (open triangles), respectively. Lower Panel: Fraction of the total number of links connected to the most connected (or "winner") node, $k_{\max } /(m t)$, plotted as a function of $\mathrm{T}$, shown for $m=2$ and for different $q$-s: $q=1.0$ (solid line), $q=1.1$ (dashed line), $q=1.3$ (dotted line), and $q=1.5$ (dot-dashed line).

emerge. This means the network experiencs a topological transition. In the whole condensate phase region ( or $T<T_{C}$ ), the power-law solution of the degree distribution hold in FGR region breaks down and hence $P(k)$ deviates from power-law distribution. However, it is still controlled by the nonextensive parameter $q$, as we can clearly see from the figure. 

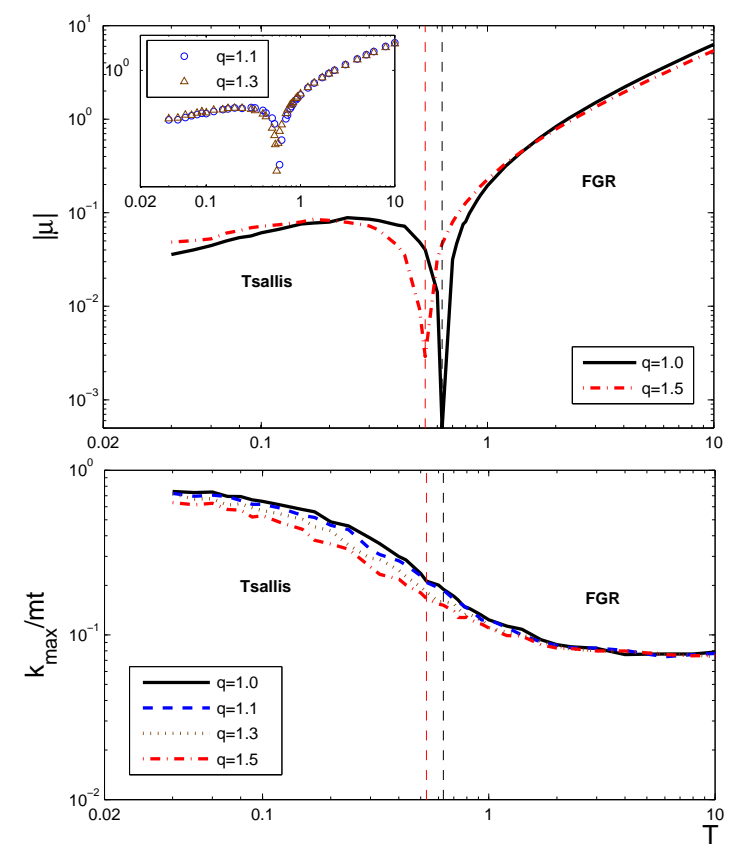

FIG. 2: Similar to Fig. 1, but simulated for energy level distribution $g(\varepsilon) \sim \sqrt{\varepsilon}$ and with different $q$-value. Upper Panel: the chemical potential $\mu$ vs. temperature $T$ with $q=1.0$ (solid line), and $q=1.5$ (dot-dashed line). The inset plot shows $q=1.1$ (open circles), and $q=1.3$ (open triangles), respectively. Lower Panel: the share of links, $k_{\max } /(m t)$ plotted as a function of $T$, shown for $m=2$ and for different $q$-value: $q=1.0$ (solid line), $q=1.1$ (dashed line), $q=1.3$ (dotted line), and $q=1.5$ (dot-dashed line), respectively.

\section{CONCLUSIONS}

In conclusion, we consider Bianconi-Barabási fitness model of growing networks in the framework of non-extensive statistical mechanics via Tsallis mapping in the fitness parameter $\eta_{i}$. We find that the phenomenological nonextensive parameter $q$ plays an important role in the dynamical evolution of complex networks: the underlying evolving networks may undergo some FGR-like phase in high temperature region with a nonextensive parameter controlled power-law degree distribution and it goes back to the original FGR phase of B-B fitness model in $q \rightarrow 1$ limit; on the other hand, in low temperature regime, particularly for $T<T_{C}$ (where $T_{C}$ is the critical temperature), a condensate phase emerges. For this condensate phase, its connectivity degree distribution $P(k)$ deviates from the power-law behavior due to the fact that the power-law solution hold in FGR region breaks down, but 

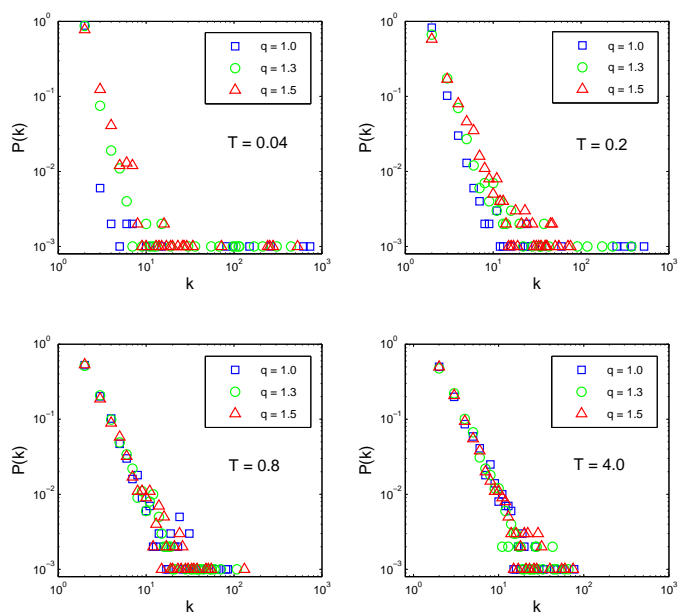

FIG. 3: The probability distribution of the degree of connectivity $P(k)$ with $q=1.0,1.3$, and 1.5 , for energy level distribution $g(\varepsilon) \sim \varepsilon$, at the different temperatures (from low to high). Upper-left Panel: at temperature $T=0.04$, and $q=1.0$ (open sqaures), $q=1.3$ (open circles), and $q=1.5$ (open triangles); Upper-right Panel: similar to the upper-left panel but at temperature $T=0.2$; Lower-left and -right Panels: similar to upper panels but at temperatures $T=0.8$ and $T=4.0$, respectively.

its specific form is controlled by the nonextensive parameter $q$. The corresponding critical transition temperature $T_{C}$ is also identified and found decrease comparing to that of the original B-B fitness model.

Tsallis formulation of nonextensive statistical mechanics not only provides a natural framework for describing the dynamical evolution of the growing complex networks, but also introduces a new complexity or entropy measure. This entropy measure is expected to be different from the traditional Shannon entropy or Gibbs entropy measures, e.g., those introduced in Refs. [32 36] in the context of complex networks. The current research opens a different angle to explore such measure, and our results may stimulate some further investigations on this regard [37].

\section{Acknowledgments}

We acknowledge to the national Natural Science Foundation of China (NSFC) for financial support, under Contract No. 10875058. The authors Y.Z. and G.S. also thank the support 
from the Initiative Plan of Shanghai Education Committee (Project No. 10YZ76) and the Scientific Research Foundation for the Returned Overseas Chinese Scholars, State Education Ministry (SRF for ROCS, SEM).

[1] D. J. Watts and S.H. Strogatz, Nature, 393 (1998) 440.

[2] A.-L. Barabási, E. Albert, Science, 286 (1999) 509; A.-L. Barabási, E. Albert, and H. Jeong, Physica (Amsterdam) 281 A (2000) 69.

[3] S. H. Strogatz, Nature, 410 (2001) 268.

[4] R. Albert and A.-L. Barabási, Rev. Mod. Phys. 74 (2002) 47.

[5] S. N. Dorogovtesev, J. F. F. Mendes, Adv. Phys. 51 (2002) 1079; M. E. J. Newman, SIAM Rev. 45 (2003) 167.

[6] D. J. Watts, Small Worlds: The Dynamics of Networks between Order and Randomness, Princeton University Press, Princeton, NJ, 1999; S. Bornholdt and H. G. Schuster (Eds.), Handbook of Graphs and Networks: From the Genome to the Internet, Wiley-VCH, Germany, 2003; R. Pastor-Satorras, A. Vespignani, Evolution and Structure of the Internet: A Statistical Physics Approach, Cambridge University Press, Cambridge, 2004.

[7] S. N. Dorogovtsev and J. F. F. Mendes, Evolution of Networks: From Biological Nets to the Internet and $W W W$, Oxford University Press, Oxford, 2003.

[8] A. V. Goltsev, S. N. Dorogovtsev and J. F. F. Mendes, Rev. Mod. Phys. 80 (2008) 1275 arXiv:0705.0010.

[9] M. J. E. Richardson, M. R. Evans, J. Phys. A 30, (1997) 6993; M. R. Evans, T. Hanney, J. Phys. A 38, (2005) R 195.

[10] P. L. Krapivsky, S. Redner and F. Leyvraz, Phys. Rev. Lett. 85, (2000) 4629.

[11] G. Bianconi and A.-L. Barabási, Phys. Rev. Lett. 86, (2001) 5632.

[12] G. Bianconi and A.-L. Barabási, Europhys. Lett. 54, (2001) 436.

[13] G. Ergün and G. J. Rodgers, Physica A 303, (2002) 261.

[14] C. Borgs, J. Chayes, C. Daskalakis and S. Roch, in Annual ACM Symposium on Theory of Computation, ACM, New York, 2007.

[15] S. N. Dorogovtsev, J. F. F. Mendes and A. N. Samukhin, Nucl. Phys. B 653, (2003) 307; Phys. Rev. Lett. 95, (2005) 195701. 
[16] P. Bialas, Z. Burda and J. Johnston, Nucl. Phys. B 493, (1996) 505; L. Borgacz, Z. Burda, W. Janke and B. Waclaw, Chaos 17, (2007) 026112.

[17] C. Godréche and J. M. Luck, Eur. Phys. Jour. B 23, (2001) 473.

[18] C. Godréche and J. M. Luck, J. Phys. A: Math. Gen. 38, (2005) 7215;

[19] J. P. Bouchaud, M. Mézard, Physica A 282, (2000) 536.

[20] J. D. Noh, G. M. Shim and H. Lee, Phys. Rev. Lett. 94, (2001) 198701; J. D. Noh, Phys. Rev. E 72, (2005) 056123.

[21] G. Palla et al., Phys. Rev. E. 69, (2004) 046117.

[22] C. Biely and S. Thurner, Phys. Rev. E 74, (2006) 066116.

[23] C. Song, S. Havlin, and H. A. Makse, Nature 433, (2005) 392.

[24] C. Tsallis, J. Stat. Phys. 52, (1988) 479.

[25] E. M. F. Curado and C. Tsallis, J. Phys. A 24 (1991) L69; ibid., 24, (1991) 3187; ibid., 25, (1992) 1019.

[26] S. R. A. Salinas and C. Tsallis (Eds.), Special Issue on Nonextensive Statistical Mechanics and Thermodynamics, Braz. J. Phys. 29, 1999.

[27] See, for example: Nonextensive Entropy - interdisciplinary applications, M. Gell-Mann and C. Tsallis (Eds.), Oxford University Press, 2004; C. Tsallis, M. Gell-Mann, Y. Sato, Extensivity and entropy production, Europhysics News 36, (2005) 186; J. P. Boon, C. Tsallis (Eds.), Nonextensive Statistical Mechanics: New Trends, New Perspectives, Europhysics News (special issue), 2005; An updated bibliography on Tsallis' nonextensive statistical mechanics can be found at http://tsallis.cat.cbpf.br/biblio.htm.

[28] D. J. B. Soares, C. Tsallis, A. M. Mariz and L. R. da Silva, Europhys. Lett. 70 (2005) 70.

[29] C. Tsallis, in Nonextensive Statistical Mechanics and Its Applications, eds. S. Abe and Y. Okamoto, Series Lecture Notes in Physics (Springer-Verlag, Heidelberg, 2001), page 3 (Appendix).

[30] K. Huang, Statistical Mechanics, 2nd. ed., Wiley (New York), 1987.

[31] Simple numerical analysis shows that the relative error of this approximation is between $10^{-3}$ and $10^{-1}$, for most values of temperature in high temperature region.

[32] D. Gfeller, J.-C. Chappelier and P. De Los Rios, Phys. Rev. E 72, (2005) 056135.

[33] L. Bogacz, Z. Burda and B. Waclaw, Physica A 366, (2006) 587.

[34] G. Bianconi, A. C. C. Coolen and C. J. Perez Vicente, Phys. Rev. E (R) 78, (2008) 016114; 
G. Bianconi, Europhys. Lett. 81, (2008) 28005; Phys. Rev. E 79, (2009) 036114.

[35] J. Gomez-Gardenes and V. Latora, Phys. Rev. E 78, (2008) 065102.

[36] F. Passerini and S. Severini, arXiv:0812.2597 (2008).

[37] G. Su, Y. Zhang, and X. Zhang, in preparation. 